\title{
How to Efficiently Predict Dengue Incidence in Kuala Lumpur
}

\author{
Duc Nghia Pham ${ }^{\S}$, Tarique Aziz ${ }^{\S}$, Ali Kohan ${ }^{\S}$, Syahrul Nellis*, Juraina binti Abd. Jamil*, Jing Jing Khoo*, Dickson Lukose ${ }^{ \pm}$, \\ Sazaly AbuBakar*, Abdul Sattar ${ }^{\#}$ and Hong Hoe Ong $\$ 1$ \\ $\S$ Artificial Intelligence Lab \\ MIMOS Berhad \\ Kuala Lumpur, Malaysia \\ * TIDREC \\ University of Malaya \\ Kuala Lumpur, Malaysia \\ ${ }^{ \pm \pm}$IIIS \\ Griffith University \\ Queensland, Australia \\ ${ }^{ \pm}$Data Science \\ GCS Agile Pty Ltd \\ Victoria, Australia
}

\begin{abstract}
Mosquito-borne diseases are rapidly spreading in all regions of the world with an estimation of 2.5 billion people globally are at risk. The recent surge in dengue outbreaks has caused severe affliction to Malaysian society. Hence, the ability to predict a dengue outbreak and mitigate its damage and loss proactively is very critical. In this paper, we study the possibility of applying machine learning (ML) and deep learning (DL) approaches to predict the number of confirmed dengue fever (DF) cases in Kuala Lumpur. We identified several contribution factors correlate to a dengue outbreak. In addition to the two frequently used factors (daily mean temperature and daily rainfall), we also took into account the enhanced vegetation index (EVI), humidity and wind speed as input factors to our prediction engines. We collected and cleansed data on these factors and the daily DF incidents in Kuala Lumpur from 2002 to 2012. We then used these data to train and evaluate our 3 ML/DL models. Among the three models, GA_RNN was the best performer and achieved a MAE of 10.95 for DF incidence prediction.
\end{abstract}

Keywords - dengue outbreak prediction, machine learning, deep learning

\section{INTRODUCTION}

Mosquito-borne diseases are rapidly spreading in all regions of the world in recent years with an estimation of 2.5 billion people globally are at risk. Among these mosquitoborne diseases, dengue is ranked as the most deadly disease in the world today [1]. Aedes mosquito species are the responsible vectors for dengue transmission. Natural and uncovered man-made rain water traps (such as ponds, blocked drains, water tanks) are the perfect oviposition and larval habitats for these mosquitoes [2].

Dengue fever (DF) can be caused by any of four dengue viral serotypes (DENV 1 - 4). Four to six days after infection, a dengue patient normally encounters a number of painful and debilitating symptoms such as sudden high fever, severe headaches, severe joint and muscle pain, nausea, fatigue, vomiting, and skin rash [3]. If a previously infected patient get contracted by a different DENV, serious problems can occur and may progress to life-threatening dengue forms called dengue hemorrhagic fever (DHF) and dengue shock syndrome (DSS) [4], [5]. The mortality rate of DHF is reportedly as high as $10-20 \%$ and it can be over $40 \%$ if DSS develops [6]. Younger children and people with weaken immune systems are at higher risk of developing DHF or DSS. Unfortunately, there is neither medicine treatment nor vaccination for dengue. People are relying on controlling Aedes mosquito species to prevent dengue outbreaks.

Consequently, dengue has been one of the main sources for hospitalisation and death (in particular for young children) and remains a major public healthcare issue for endemic countries worldwide. Resource-poor regions in the Americas, western Pacific, eastern Mediterranean, Africa and South-East Asia are highly vulnerable to transmission of dengue disease [1]. In fact, dengue incidence recorded a 30 -fold increase in the last 50 years. Worldwide, 2.5 billion people from over 100 endemic countries and areas are estimated to be vulnerable to dengue infection by the World Health Organization (WHO). Up to 50 million dengue infections occur every year, of which 500,000 DHF cases and 22,000 deaths mainly among children are reported [1]. Only 9 countries in the world experienced DHF cases prior to 1970 . Unfortunately, this number has amplified four times more since then and continues to increase.

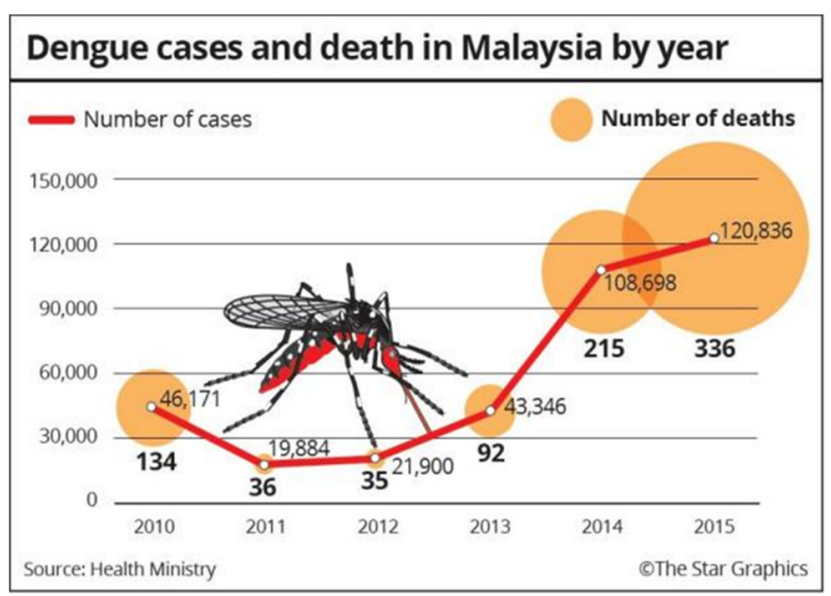

Fig. 1. Dengue cases and death in Malaysia by year ${ }^{2}$

As shown in Figure 1, the number of deaths by dengue in Malaysia in 2015 is 10 times higher than in 2012, whereas the number of infected cases in 2015 is 6 times higher than in

\footnotetext{
${ }^{1}$ Tarique Aziz, Ali Kohan and Syahrul Nellis were with MIMOS and
} TIDREC when this study was conducted. 
2012. Therefore, the ability to effectively predict potential dengue outbreaks and their sizes (i.e. the number of dengue incidents) is highly required. It will provide public health agencies ample time to properly plan, prevent and mitigate; especially for places in which hospitals and medical clinics may not cope well during an outbreak. Different contribution factors to dengue infections and outbreaks have been identified and discussed in the literature, for example the environmental factors affecting mosquito populations [2], [7], [8]. However, these research works mostly focused on the detection and surveillance of dengue outbreak, rather than proactively predicting it [9]. A recent literature review of decision support systems for control and prevention of vectorborne diseases [10] also found that none of the works reviewed proposed how to predict or forewarn of a dengue outbreak, despite lengthy identification and discussion of useful risk maps.

This paper investigates three different machine learning (ML) and deep learning (DL) approaches for prediction of dengue incidence in Kuala Lumpur (KL). We identified several contribution factors correlate to a dengue outbreak. In addition to the two frequently used factors (daily mean temperature and daily rainfall), we also took into account the enhanced vegetation index (EVI), humidity and wind speed as input factors to our prediction engines. We collected and cleansed data on these factors and the daily dengue incidents in Kuala Lumpur from 2002 to 2012. We then used these data to train and evaluate our $3 \mathrm{ML} / \mathrm{DL}$ models. We experimentally demonstrated that our GA RNN model could predict the number of confirmed DF incidents in KL area 15 days in advance with a mean absolute error of 10.95 .

\section{RELATED WORK}

Statistical analysis approach, in particular regression methods, has been widely used in many studies described in the literature for dengue outbreak prediction. A Poisson multivariate regression model was proposed to predict weekly dengue incidents in Singapore [11]. The model was trained using previous dengue incidents during 2000-2010 as well as weekly average temperature and cumulative rainfall during that period. The model was then validated on observed dengue incidents and weather data from 2011 to 2012. Another application of statistical analysis to forecast dengue outbreaks was proposed in [12]. Two environment-based multivariate, autoregressive models was developed to forecast dengue outbreaks in Cali, Colombia from 2 weeks to 6 months in advance using epidemiological and meteorological data in the period from 2000 to 2011 . Significant dengue outbreaks was found to occur generally during warm-and-dry $\left(18^{\circ} \mathrm{C}\right.$ to $\left.32^{\circ} \mathrm{C}\right)$ periods. ARIMA and SARIMA models, which were widely used for financial prediction due to its special consideration of the seasonality of financial data, were also often used for dengue outbreak prediction [5], [13], [14]. However, the environmental conditions (regularly used for dengue prediction) are less likely to follow regular patterns due to climate change.

Yu, Yang, Yen \& Christakos [15] used a more diverse dataset of human health data, rainfall, temperature, Southern Oscillation Index, and mosquito larvae abundance collected in Taiwan between 2002 and 2006 to train their Bayesian Maximum Entropy model. They then validated its prediction for 1 week in advance using the 2007 data. They found that the probabilities that a dengue outbreak actually occurred given a positive/negative prediction were 0.5541 and 0.031 , respectively. Lowe et al. [16] combined a spatio-temporal hierarchical model with a Bayesian framework using Markov Chain Monte Carlo into a Bayesian generalized linear mixed model. They used this model to predict monthly dengue incidents in Brazil and achieved improvement both spatially and temporally.

Rule-based techniques have recently been applied to enhance the prediction of dengue outbreaks [17], [18] in the cases of nonlinear models and high dimensional data which may contain lots of interacting parameters. These techniques automatically discover the correlation between input parameters and the predicted outcomes and generate association rules to represent these relationships. Users are required to carefully set a pre-determined criteria of significance to filter out noisy or anomaly association rules, and thus excluding all weakly correlated variables. The final set of rules is then build into a rule classifier for prediction. It is worthy to note that [17], unlike other studies, used medical dengue records in Selangor, Malaysia as the main data source to predict whether a dengue infected patient was a repeated case, rather than predicting the number of infections. A key disadvantage of rule-based methods is their inability to predict the actual number of infections and can only predict whether the dengue incidence will be in High, Medium, or Low categories.

\section{CONTRibution Factors to A Dengue OUtBreaK}

Previous studies have identified several contribution factors that are related to a dengue outbreak. These data parameters for predicting a dengue outbreak that were identified and discussed in the literature can broadly be categorised in into two groups: (i) environmental data, and (ii) epidemiological \& socioeconomic data.

\section{A. Environmental Data}

It is well known that dengue is transmitted by infected Aedes mosquitoes. Research has showed that environmental conditions can heavily impact the life cycle of these mosquito species. Thus, the ability to perform weather forecast and predict its suitability for the survival and viral transmission of Aedes mosquitoes are very critical to the success of a dengue outbreak prediction model. Table 1 summarises different types of environmental parameters known to have significant correlation with dengue incidence.

TABLE I. ENVIRONMENTAL CONTRIBUTION FACTORS

\begin{tabular}{|l|l|}
\hline Rainfall & Temperature \\
\hline Altitude & Biome \\
\hline Land surface temperature & Remotely sensed vegetation \\
\hline Sea surface temp anomalies & Southern Oscillation Index \\
\hline Humidity & Mosquito population index \\
\hline Larvae population index & \\
\hline
\end{tabular}

\section{B. Epidemiological and Socioeconomic Data}

Dengue fever is transmitted from an infected person to a healthy one by the bite of an Aedes mosquito infected with the virus. Thus, it is essential to take into account the historical number of dengue infection cases as it reflects the virus presence rate in the local population. 
Furthermore, there are many good socioeconomic predictors for dengue prediction [19]. Indeed, data on people movement and population changes can help predict the virus presence's variation. Southeast Asian (SEA) countries are particularly vulnerable to dengue infection. Recent studies showed that an increase of international travel is the main driver of global transmission and expansion of these mosquito-borne diseases. In particular, immigrants or tourists from endemic countries travel to another country and spread of vectors into new ecological niches. The increasing availability of budget flights in SEA nations further boost the mobility of SEA population and consequently the vulnerability of the region to mosquito-borne diseases.

Other socioeconomic parameters such as the availability of electricity and drinking water, as well as poverty index are also good indicators because of their reflection on people's living conditions. Useful epidemiological and socioeconomic parameters for dengue incidence prediction are summarised in Table 2.

TABLE II. EPIDEMIOLOGICAL \& SOCIOECONOMIC CONTRIBUTION FACTORS

\begin{tabular}{|l|l|}
\hline Dengue incidence & Census (population) data \\
\hline Administrative boundaries & Poverty index \\
\hline Electricity access & Drinking water access \\
\hline Sanitation index & Child health index \\
\hline Child education index & Child quality of life index \\
\hline Human population movement & Vector control \\
\hline
\end{tabular}

\section{OUR APPROACH}

\section{A. Data Collection and Cleansing}

This paper focused on predicting dengue incidence in the Kuala Lumpur city area. The daily dengue incident records in this area for the years 2002-2012 were provided by the Kuala Lumpur City Council. They represents how many confirmed dengue incidents were reported every day in Kuala Lumpur. Unfortunately these daily incident data were too noisy. For example, the total number of cases for a particular month may be high but the turnout for a few days within that month may still low due to different social and economic reasons. People, even being sick, tends to visit the hospital on certain days due to convenience. To overcome this issue, we used the averages of these data over a period of time as input. We found that 15days interval (coincidently equals to the EVI interval) produces the most accurate predictions. We were not able to obtain data for the other 11 out of 12 epidemiological and socioeconomic factors identified above.

For environmental data, we managed to obtain data covered Kuala Lumpur area for the period of 2002-2012 for five contribution factors out of 11 environmental factors identified in Table 1 . The first four factors (daily rainfall, daily temperature, daily humidity, and daily wind speed) were the Malaysian Meteorological Department. Those data came from four different weather stations that cover Kuala Lumpur area: FRI Kepong, Klang Gate, Parliament, and Petaling Jaya. Missing data points were interfered using cubic spline interpolation. For each of these four environmental factors, we then averaged out its data from all stations to obtain a single input parameter.

We obtained data for EVI from NASA as the last environment factor used in this study. EVI data were provided for each zone of $500 \mathrm{~m}^{2}$ land area every 15 days. Again, cubic spline interpolation was used to interfere the missing data. We downloaded EVI data from all the small zones (of $500 \mathrm{~m}^{2}$ ) covering Kuala Lumpur and then aggregated them into 16 bigger zones. Thus, there is a total of 16 EVI input parameters.

In summary, 21 different input parameters were used in study (as shown in Table 3 ).

TABLE III. INPUT PARAMETERS.

\begin{tabular}{|l|l|l|l|}
\hline No. & Parameter & No. & Parameter \\
\hline 1 & Dengue incidence & 12 & EVI (zone 7) \\
\hline 2 & Rainfall & 13 & EVI (zone 8) \\
\hline 3 & Temperature & 14 & EVI (zone 9) \\
\hline 4 & Humidity & 15 & EVI (zone 10) \\
\hline 5 & Wind speed & 16 & EVI (zone 11) \\
\hline 6 & EVI (zone 1) & 17 & EVI (zone 12) \\
\hline 7 & EVI (zone 2) & 18 & EVI (zone 13) \\
\hline 8 & EVI (zone 3) & 19 & EVI (zone 14) \\
\hline 9 & EVI (zone 4) & 20 & EVI (zone 15) \\
\hline 10 & EVI (zone 5) & 21 & EVI (zone 16) \\
\hline 11 & EVI (zone 6) & & \\
\hline
\end{tabular}

\section{B. Prediction Models}

1) Genetic Algorithm enhanced Recurrent Neural Network (GA_RNN)

With the rapid evolution of general-purpose graphics processing units (GPGPU), deep learning has helped advancing the state-of-the-art of solving problems in computer vision, autonomous driving, etc. Recurrent neural network based methods have also been demonstrated to give satisfactory performances compared to statistical analysis methods (e.g. ARIMA) in time series prediction [20]-[22]. In this study, we adapted the long short-term memory (LSTM) method [23] to predict dengue incidence.

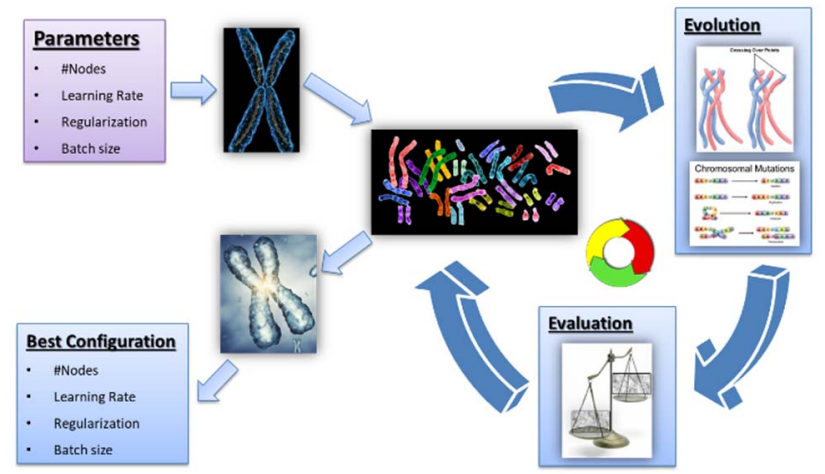

Fig. 2. GA process to optimise LSTM model configuration

It is well known that the performance of LSTM (and other deep learning methods) is greatly dependent on the carefully crafting of several parameters such as the number of nodes, learning rate, regularization, batch size, etc. Our initial experiments also confirmed this drawback. Hence, we decided to use genetic algorithm (GA) [24] to optimise LSTM model's configuration. Here we configured a chromosome to represent the values of these LSTM parameters: the number of nodes, learning rate, 
regularization, batch size. Figure 2 depicts the process to optimise the model configuration of LSTM. Algorithm 1 shows the pseudo-code of our GA_RNN method.

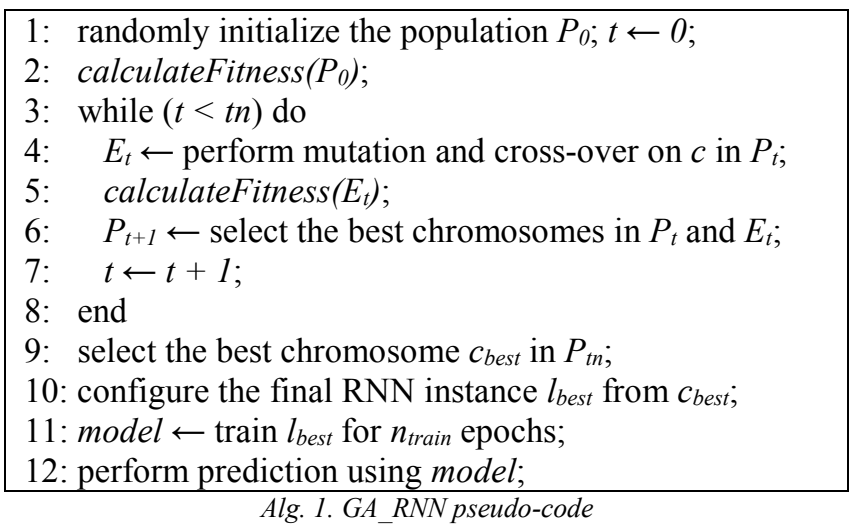

Lines 1-8 in Algorithm 1 describe how we used GA to optimise the model configuration for our RNN, in particular an LSTM network. It starts with a random generated population of $n$ chromosomes, each encodes a particular configuration (i.e. parameter values) for our LSTM network. At each iteration $t$, mutation and cross-over operators are applied on half of the population $P_{t}$ to evolve a new set of chromosomes $E_{t}$. Algorithm 2 describes how to evaluate the fitness of a chromosome $c$ by configuring an LSTM instance $l$ with parameter values encoded in $c$ and training $l$ for $n_{\text {eval }}$ epochs. The fitness of $c$ is defined as the mean absolute error (MAE) of $l$ on training results. The new population $P_{t+l}$ is created by selecting the $n$ best chromosomes from $P_{t}$ and $E_{t}$ (line 6 in Algorithm 1). The final LSTM network $l_{\text {best }}$ is configured using settings from the best chromosome $c_{\text {best }}$ (lines 9-10). It is then trained to generate the model for dengue prediction (lines 11-12).

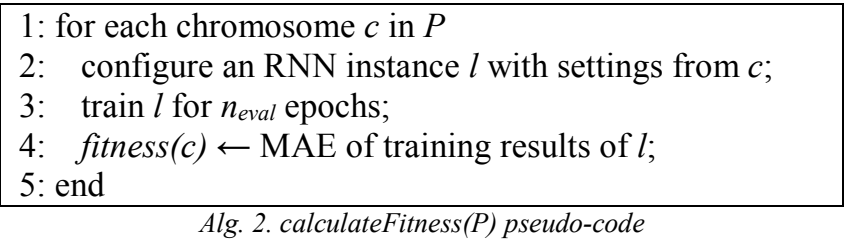

\section{2) Linear Regression (LR)}

In our previous study [25], we developed a simple LR model with 3 parameters (EVI, daily rainfall and temperature) to predict dengue cases. Our experiments on data collected within a $15 \mathrm{~km}$ radius area from University of Malaya Medical Centre was promising. Therefore, we extended this LR model to incorporate all the contribution factors identified in section 4A. Like the previous small dataset, no regular seasonal pattern was found in the dataset used in this study. LR remains a good choice as it can deal with irregular observable variables and produces model with low variances.

Given $n$ data factors $\left(f_{1}, f_{2}, \ldots, f_{n}\right)$, our linear regression model can be formally defined as:

$$
F(d)=C+a_{1} * f_{1}+a_{2} * f_{2}+\ldots+a_{n} * f_{n}
$$

- $F$ : the dengue incidence transformation function

- $d$ : the dengue incidence rate

- $C$ : a constant factor

$-a_{1}, a_{2}, \ldots, a_{n}$ : the coefficients for factors $f_{1}, f_{2}, \ldots, f_{n}$

\section{3) Decision Tree (DT)}

As rule-based approach is also good in predicting the high and low ranges of dengue incidence, we adapted a DT method to approximately predict DF cases. Rather than predict the actual daily cases, our DT works out the value ranges of historical daily cases from the training set and generate rules to predict which range the daily incidents will be in.

\section{EXPERIMENTAL RESULTS}

We implemented the GA_RNN method with Theano ${ }^{3}$ and DEAP ${ }^{4}$ libraries. Our LR method was coded with the GNU Octave language ${ }^{5}$ and our DT method was coded in the BigML platform ${ }^{6}$. Note that our decision to select these language, platform and libraries was purely to shorten the implementation cycle. One drawback from selecting BigML was that we were able to capture only the statistics of the test results of DT model but not its detailed results.

For all the experiments conducted in this study, we used data from 2002-2010 as training dataset and data from 20112012 as test dataset. We first run GA to optimise the configuration of RNN. Figure 3 shows how the fitness function value changed over 70 generation cycles. Table 4 shows the best RNN configuration after 70 generations.

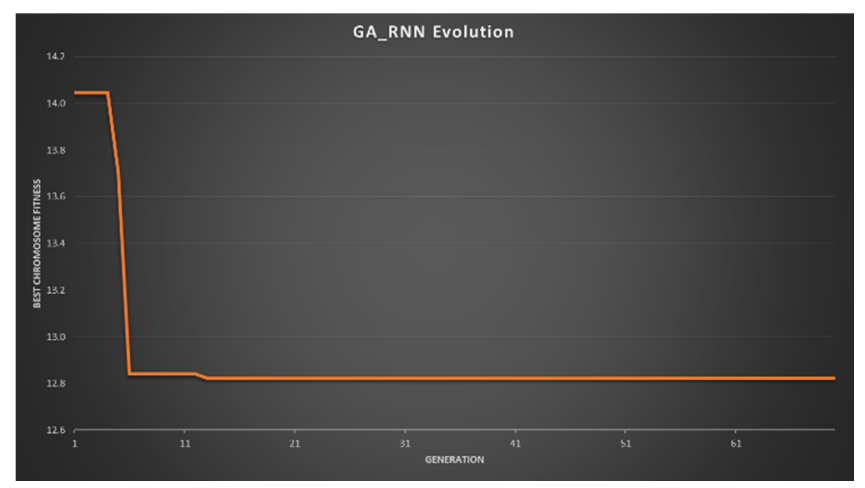

Fig. 3. Fitness values of RNN models over GA generation cycles.

TABLE IV. THE BEST CONFIGURATION FOR RNN TRAINED BY GA

\begin{tabular}{|c|c|c|c|}
\hline \#nodes & Learning rate & Regularization & Batch size \\
\hline 945 & 0.00004 & 0.0001 & 30 \\
\hline
\end{tabular}

We then trained our three models with the 2002-2010 training dataset. Figures 4 and 5 show the scatter plot of the trained outcomes of GA_RNN and LR against the actual cases from our training set. The linear trend lines of GA_RNN (in blue) and LR (in yellow) are also shown in these figures.

where:

\footnotetext{
${ }^{3} \mathrm{http}: / /$ deeplearning.net/software/theano/

${ }^{4} \mathrm{https}: / /$ github.com/DEAP/deap
}

${ }^{5} \mathrm{https}: / /$ www.gnu.org/software/octave/
${ }^{6} \mathrm{https}: / /$ bigml.com/ 


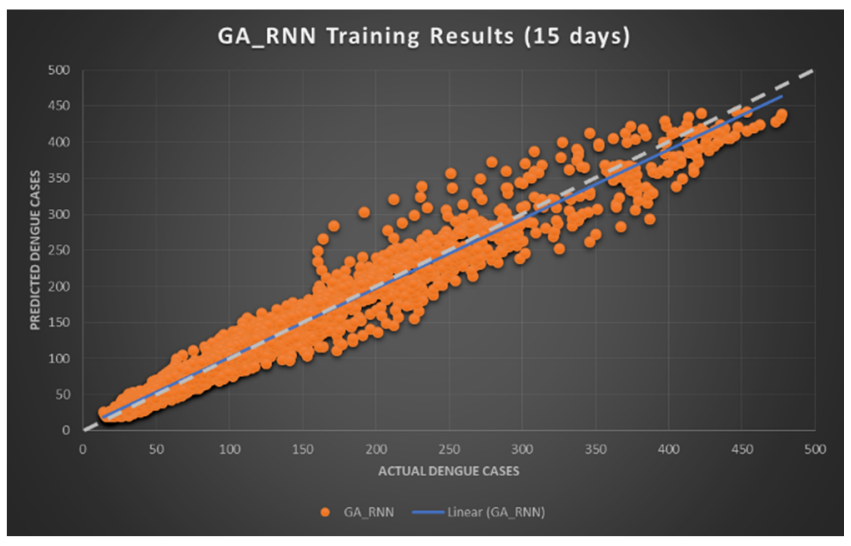

Fig. 4. Scatter plot of GA_RNN outputs versus the actual number of cases from training dataset.

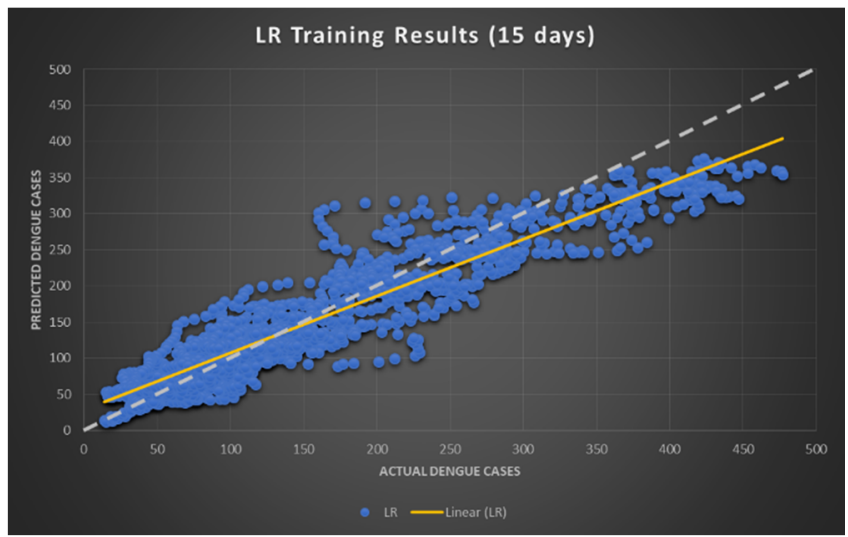

Fig. 5. Scatter plot of LR outputs versus the actual number of cases from training dataset

As shown in Fig. 4 and 5, the training results of GA RNN align better than those of LR when comparing against the actual number of dengue incidents from the training set. During training, the predicted results by GA_RNN closely matched the historical training values, while LR produced values that were likely lower than the historical values. This observation is further supported by looking at the linear trend lines of both models: GA_RNN's trend line almost overlaps with the main diagonal (Fig. 4) while LR's trend line goes lower and lower away from the main diagonal (Fig. 5).

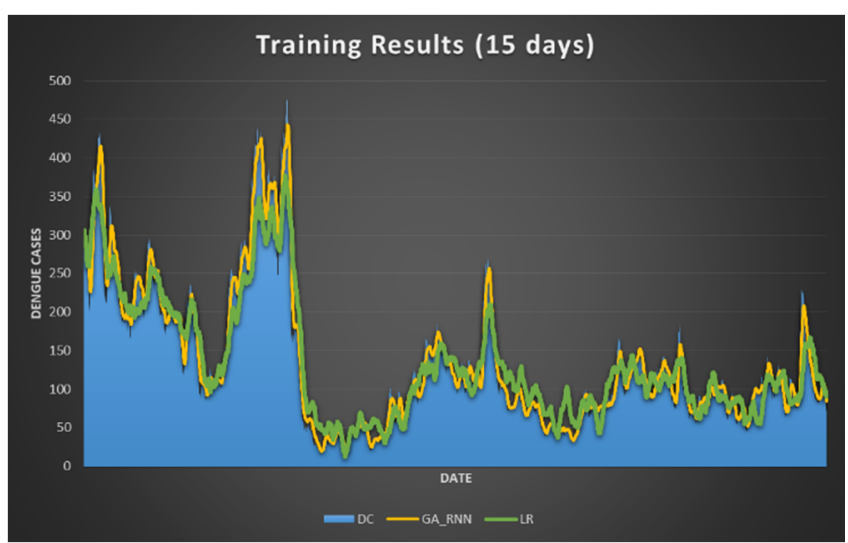

Fig. 6. GA_RNN and LR outputs versus the actual number of cases $(D C)$ from training dataset.

Figure 6 plots the same training results of both models, GA RNN (yellow line) and LR (green line), against the historical dengue cases (in light blue area) across the time line from 2002 to 2010 on a daily basis. This graph confirms our observation from the scatter plots in Fig. 4 and 5: GA_RNN's training results fit closely to the actual curve while the results of LR tends to shift further into the future.

Finally, we ran the three trained models (GA_RNN, LR and DT) on our test dataset for dengue incidence prediction 15 days in advance. In order to measure the performance of our models against the actual number of cases, we calculated and reported the mean absolute errors (MAEs) and the root mean square errors (RMSEs) of these models in Figure 7. Out of the three models, GA_RNN produced the most accurate prediction (with a MAE of 10.95 and an RMSE of 13.06) and DT was the worst performer, producing results almost three times higher in error statistics than GA_RNN (with a MAE of 25.32 and an RMSE of 34.86).

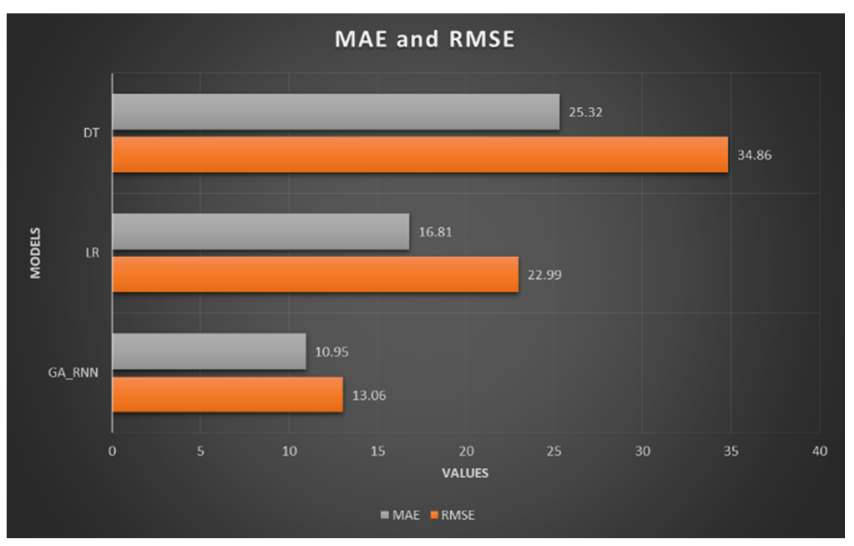

Fig. 7. Mean absolute errors (MAEs) and Root mean square errors (RMSEs) of the predicted dengue cases (15 days in advance) of GA_RNN and $L R$.

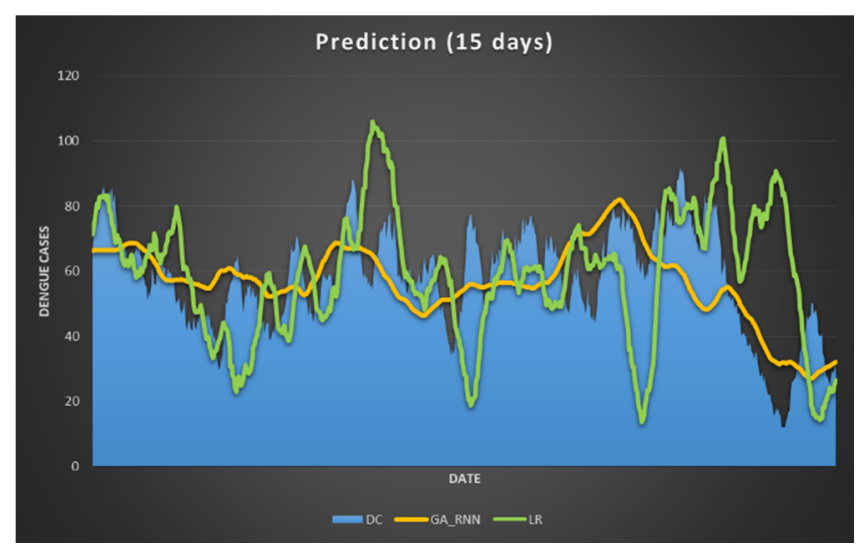

Fig. 8. Predicted dengue cases (15 days in advance) of GA RNN and LR versus the actual number of cases (DC) from the test dataset.

Figure 8 plots the predicted dengue cases from GA_RNN and LR against the actual cases from our test dataset across the time line from 2011 to 2012 on a daily basis. Again, the results of DT were omitted in Fig. 8 as we weren't able to capture its detailed test results. LR's prediction curve closely resembled the curve of the actual dengue incidence during the test period, but its prediction was even more offset to the future than its curve produced during training (as shown in Fig. 6). Though the prediction was more accurate in preserving peaks and off-peaks of dengue cases, it fell in the future to the actual dates, which made this result not useful for taking pro-active actions to prevent the spread of dengue virus. On the other hand, although the predicted curve of 
GA_RNN was less closely resemble to the actual curve and appeared to be smoother, its predicted cases followed the trend of the actual occurrence and were much closer to the actual dates of the occurrences. This made the results produced by GA_RNN more useful for taking necessary preventive action to mitigate the spread of dengue virus.

\section{CONCLUSION}

In conclusion, we proposed and benchmarked three different ML/DL models for predicting the confirmed DF incidence 15 days in advance. We found that GA_RNN was the best performer, following by LR. The worst performance of DT was expected as it could only predict the value bucket that the future dengue incidence would most likely be in and then reported the median of that bucket, rather than predicting the actual value. We also found that the predicted curve of LR highly matched the actual incidence curve even though its curve was shifted into future and its error statistics came second among the three benchmarked models. In future we plan to improve the performance of LR model by reducing its right shifting effect.

\section{ACKNOWLEDGMENT}

This research was supported by the Science Fund Grant 01-03-04-SF0061 from the Ministry of Energy, Science, Technology, Environment and Climate Change (MESTECC).

\section{REFERENCES}

[1] WHO. (2015) Impact of dengue. [Online]. Available: http://www.who.int/csr/disease/dengue/impact/en/.

[2] D. A. Focks, E. Daniels, D. Haile, and J. Keesling, "A simulation model of the epidemiology of urban dengue fever: literature analysis, model development, preliminary validation, and samples of simulation results." The American journal of tropical medicine and hygiene, vol. 53 5, pp.489-506, 1995.

[3] D. J. Gubler, "Dengue and dengue hemorrhagic fever," Clinical microbiology reviews, vol. 11 , no. 3, pp. 480-496, 1998

[4] P. Avirutnan, N. Punyadee, S. Noisakran, C. Komoltri, S. Thiemmeca, K. Auethavornanan, A. Jairungsri, R. Kanlaya, N Tangthawornchaikul, C. Puttikhunt, S.-n. Pattanakitsakul, P.-t. Yenchitsomanus, J. Mongkolsapaya, W. Kasinrerk, N. Sittisombut, M. Husmann, M. Blettner, S. Vasanawathana, S. Bhakdi, and P. Malasit, "Vascular leakage in severe dengue virus infections: A potential role for the nonstructural viral protein ns1 and complement," The Journal of Infectious Diseases, vol. 193, no. 8, pp. 1078-1088, 2006. [Online]. Available: http://dx.doi.org/10.1086/500949

[5] S. Bhatnagar, V. Lal, S. D. Gupta, and O. P. Gupta, "Forecasting incidence of dengue in Rajasthan, using time series analyses." Indian journal of public health, vol. 56 4, pp. 281-5, 2012.

[6] R. V. Gibbons and D. W. Vaughn, "Dengue: an escalating problem," $B M J$, vol. 324, no. 7353, pp. 1563-1566, Jun 2002.

[7] P. Barbazan, M. Guiserix, W. Boonyuan, W. Tuntaprasart, D. Pontier, and J.-P. Gonzalez, "Modelling the effect of temperature on transmission of dengue," Medical and Veterinary Entomology, vol. 24, no. 1, pp. 66-73.

[8] C.-S. Shang, C.-T. Fang, C.-M. Liu, T.-H. Wen, K.-H. Tsai, and C.-C. King, "The role of imported cases and favorable meteorological conditions in the onset of dengue epidemics," in PLOS Neglected Tropical Diseases, 2010.

[9] S. Runge-Ranzinger, O. Horstick, M. Marx, and A. Kroeger, "What does dengue disease surveillance contribute to predicting and detecting outbreaks and describing trends?" Tropical medicine and international health, vol. 13 8, pp. 1022-41, 2008.
[10] L. Eisen and R. J. Eisen, "Using geographic information systems and decision support systems for the prediction, prevention, and control of vector-borne diseases," Annual Review of Entomology, vol. 56, no. 1, pp. 41-61, 2011, pMID: 20868280. [Online]. Available: https://doi.org/10.1146/annurev-ento-120709-144847

[11] Y. L. Hii, H. Zhu, N. Ng, L. C. Ng, and J. Rocklv, "Forecast of dengue incidence using temperature and rainfall," PLOS Neglected Tropical Diseases, vol. 6, no. 11, pp. 1-9, 11 2012. [Online]. Available: https://doi.org/10.1371/journal.pntd.0001908

[12] M. D. Eastin, E. Delmelle, I. Casas, J. Wexler, and C. Self, "Intra- and interseasonal autoregressive prediction of dengue outbreaks using local weather and regional climate for a tropical environment in Colombia," The American Journal of Tropical Medicine and Hygiene, vol. 91, no. 3, 2014.

[13] N. C. Dom, A. A. Hassan, Z. A. Latif, and R. Ismail, "Generating temporal model using climate variables for the prediction of dengue cases in Subang Jaya, Malaysia," 2013.

[14] M. Gharbi, P. Quenel, J. Gustave, S. Cassadou, G. L. Ruche, L. Girdary, and L. Marrama, "Time series analysis of dengue incidence in Guadeloupe, French West Indies: Forecasting models using climate variables as predictors," BMC Infectious Diseases, vol. 11, no. 1, p. 166, Jun 2011. [Online]. Available: https://doi.org/10.1186/14712334-11-166

[15] H.-L. Yu, S.-J. Yang, H.-J. Yen, and G. Christakos, "A spatio-temporal climate-based model of early dengue fever warning in Southern Taiwan," Stochastic Environmental Research and Risk Assessment, May 2011.

[16] R. Lowe, T. C. Bailey, D. B. Stephenson, R. Graham, C. A.S. Coelho, M. Carvalho, and C. Barcellos, "Spatio-temporal modelling of climate sensitive disease risk: Towards an early warning system for dengue in Brazil," Computers \& Geosciences, vol. 37, no. 3, pp. 371-381, March 2011.

[17] A. A. Bakar, Z. Kefli, S. Abdullah, and M. Sahani, "Predictive models for dengue outbreak using multiple rulebase classifiers," in Proceedings of the 2011 International Conference on Electrical Engineering and Informatics, July 2011, pp. 1-6.

[18] A. L. Buczak, P. T. Koshute, S. M. Babin, B. H. Feighner, and S. H. Lewis, "A data-driven epidemiological prediction method for dengue outbreaks using local and remote sensing data," BMC Medical Informatics and Decision Making, vol. 12, no. 1, p. 124, Nov 2012. [Online]. Available: https://doi.org/10.1186/1472-6947-12-124

[19] C. Åström, J. Rocklöv, S. Hales, A. Béguin, V. Louis, and R. Sauerborn, "Potential distribution of dengue fever under scenarios of climate change and economic development," EcoHealth, vol. 9, no. 4, pp. 448-454, Dec 2012. [Online]. Available: https://doi.org/10.1007/s10393-012-0808-0

[20] S. Ho, M. Xie, and T. Goh, "A comparative study of neural network and box-jenkins ARIMA modeling in time series prediction," Computers and Industrial Engineering, April 2002.

[21] J. Zhang and K. F. Man, "Time series prediction using mn in multidimension embedding phase space," in IEEE International Conference on Systems, Man, and Cybernetics, 1998.

[22] Z. Che, S. Purushotham, K. Cho, D. A. Sontag, and Y. Liu, "Recurrent neural networks for multivariate time series with missing values," in Scientific Reports, 2018.

[23] S. Hochreiter and J. Schmidhuber, "Long short-term memory," Neural Computation, vol. 9, no. 8, pp. 1735-1780, Nov. 1997. [Online]. Available: http://dx.doi.org/10.1162/neco.1997.9.8.1735

[24] D. Whitley, "A genetic algorithm tutorial," Statistics and Computing, vol. 4, no. 2, pp. 66-85, June 1994.

[25] D. N. Pham, T. Aziz, A. Kohan, S. Nellis, J. binti Abd. Jamil, J. J. Khoo, D. Lukose, S. AbuBakar, and A. Sattar, "An efficient method to predict dengue outbreaks in kuala lumpur," in Proceeding of the $3 r d$ International Conference on Artificial Intelligence and Computer Science, 2015. 